\title{
Erratum to: Infusion of donor lymphocytes expressing the herpes simplex virus thymidine kinase suicide gene for recurrent hematologic malignancies after allogeneic hematopoietic stem cell transplantation
}

\author{
Hisayoshi Hashimoto ${ }^{1}$ Shigehisa Kitano ${ }^{2,3} \cdot$ Ryosuke Ueda $^{1} \cdot$ Ayumu Ito $^{4} \cdot$ Kohei Tada $^{3,4}$. \\ Shigeo Fuji ${ }^{4}$ Sung-Won Kim ${ }^{4}$ 'Takuya Yamashita ${ }^{4}$ Daisuke Tomura ${ }^{5}$ Ikuei Nukaya ${ }^{5}$. \\ Junichi Mineno $^{5} \cdot$ Takahiro Fukuda $^{4} \cdot$ Shinichiro Mori $^{6} \cdot$ Yoichi Takaue $^{7} \cdot$ Yuji Heike $^{8}$ \\ Published online: 11 September 2015 \\ (c) The Japanese Society of Hematology 2015
}

\section{Erratum to: Int J Hematol (2015) 102:101-110 DOI 10.1007/s12185-015-1801-5}

The corresponding author of the article would like to add "Sung-Won Kim" in the author group. The revised author group is as follows:

Hisayoshi Hashimoto - Shigehisa Kitano - Ryosuke Ueda · Ayumu Ito · Kohei Tada - Shigeo Fuji · Sung-Won Kim · Takuya Yamashita · Daisuke Tomura - Ikuei Nukaya . Junichi Mineno - Takahiro Fukuda - Shinichiro Mori . Yoichi Takaue · Yuji Heike

The online version of the original article can be found under doi:10.1007/s12185-015-1801-5.

Yuji Heike

heiyuji@luke.ac.jp

1 Division of Molecular and Cellular Medicine, National Cancer Center Research Institute, 5-1-1 Tsukiji, Chuo-ku, Tokyo 104-0045, Japan

2 Division of Experimental Therapeutics, National Cancer Center Hospital, 5-1-1 Tsukiji, Chuo-ku, Tokyo 104-0045, Japan

3 Division of Cancer Immunotherapy, Exploratory Oncology Research and Clinical Trial Center, National Cancer Center, 5-1-1 Tsukiji, Chuo-ku, Tokyo 104-0045, Japan

4 Division of Hematopoietic Stem Cell Transplantation, National Cancer Center Hospital, 5-1-1 Tsukiji, Chuo-ku, Tokyo 104-0045, Japan
5 Center for Cell and Gene Therapy, Takara Bio Inc, Seta 3-4-1, Otsu, Shiga 520-2193, Japan

6 Division of Hematology and Oncology, St. Luke's International Hospital, 9-1 Akashi-cho, Chou-ku, Tokyo 104-8560, Japan

7 Research Planning and Management Department, St. Luke's International Hospital, 9-1 Akashi-cho, Chou-ku, Tokyo 104-8560, Japan

8 Immunotherapy and Cell Therapy Service, St. Luke's International University and Hospital, 9-1 Akashi-cho, Chou-ku, Tokyo 104-8560, Japan 